ارزيابى تنوع زنتيكى و ساختارشناسى تودههاى خربزه وحشى (Cucumis melo var. agrestis) حاشيه جنوبى

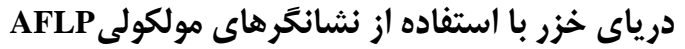

\begin{tabular}{|c|}
\hline 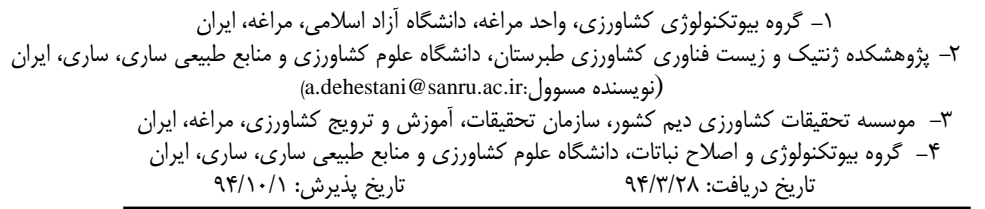 \\
\hline
\end{tabular}

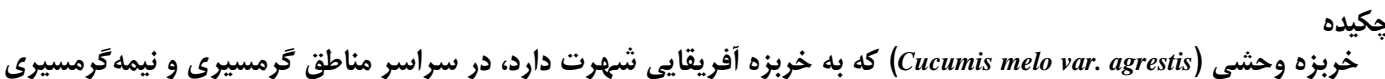

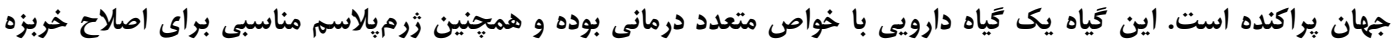

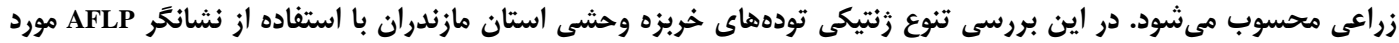

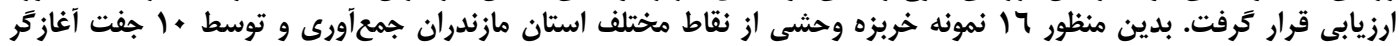

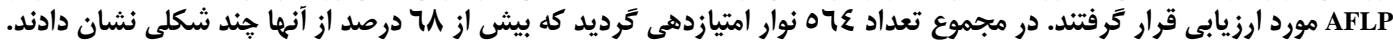

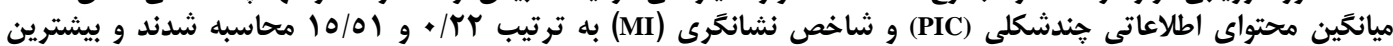

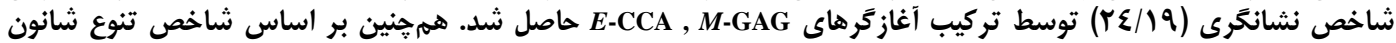

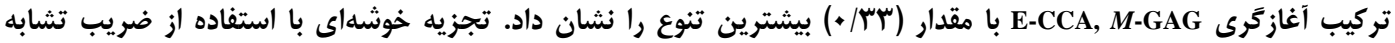

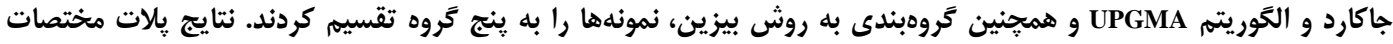

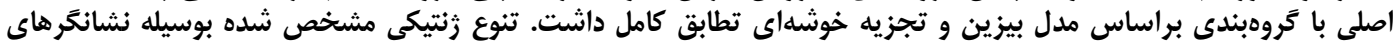

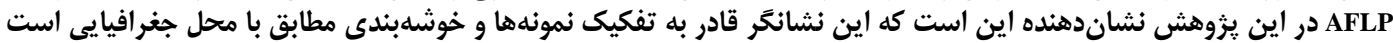

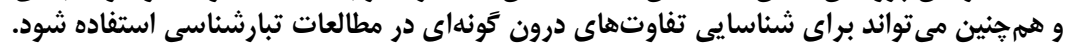

وازههاى كليدى: تنوع زنتيكى، خربزه وحشى، روش بيزين، نشانكَر AFLP

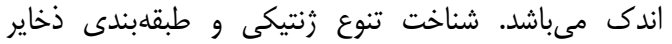

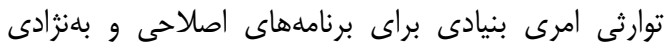

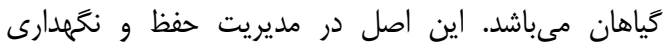

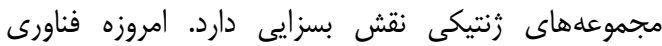

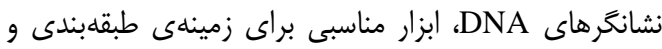

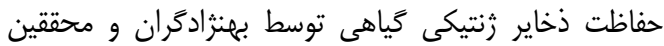

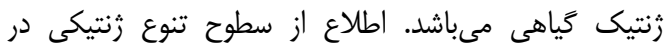

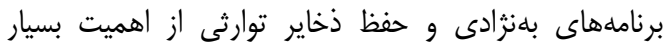

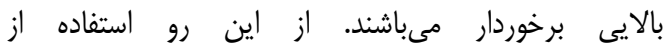

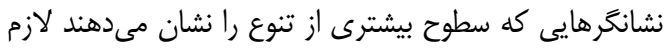

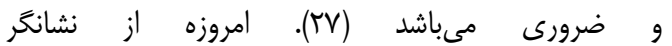
(Amplified fragment length polymorphism) AFLP

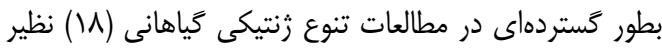

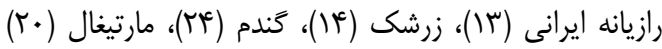

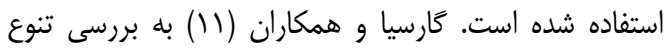

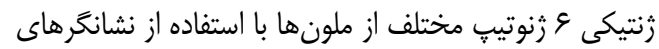

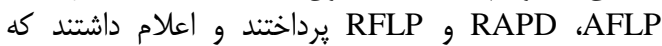
نشانكر AFLP كارايى بالاترى نسبت به ساير نشانكر ها داشته است. نشانكر AFLP در سال 1990 توسط وُس وُس وهمكاران

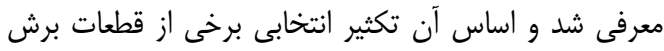

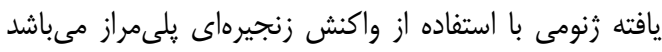

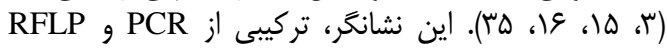

مقدمه

خربزه وحشى (Cucumis melo var. agrestis) متعلق

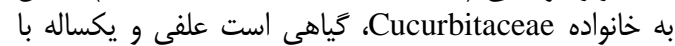

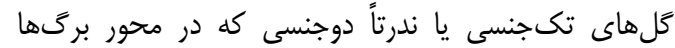

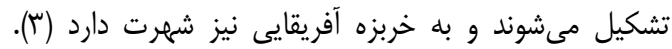

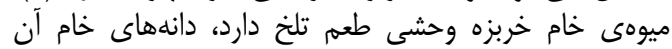

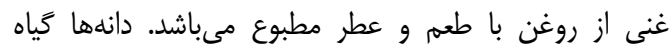

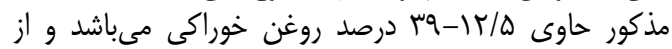

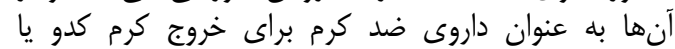

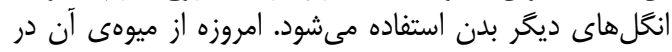

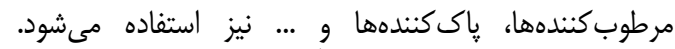

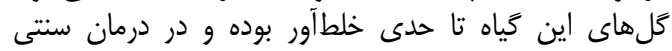

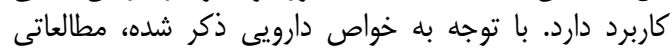

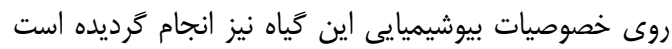

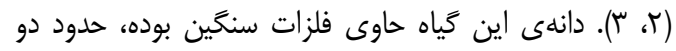

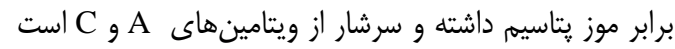

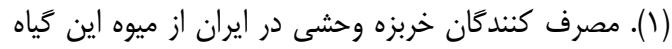

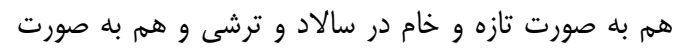

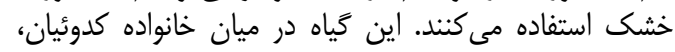

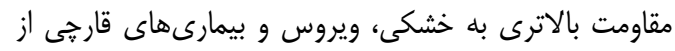

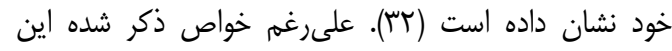
كياه، مطالعات تنوع زنتيكى بخصوص در شمال كشور بسيار 


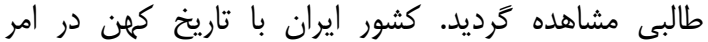

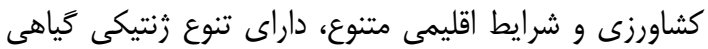

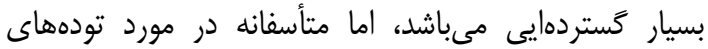

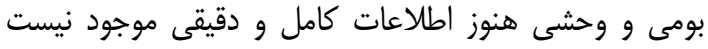

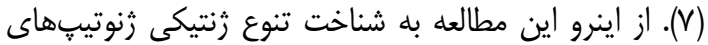

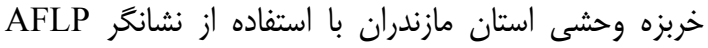

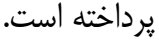

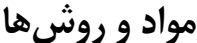

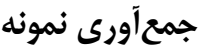

اين تحقيق در يزوهشكده زئن زنتيك و زيست فناورى

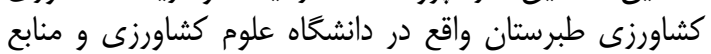

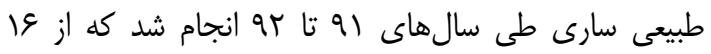

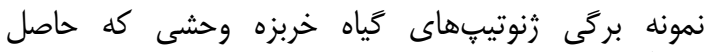

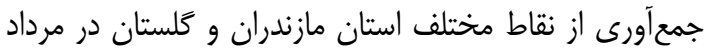

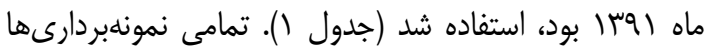

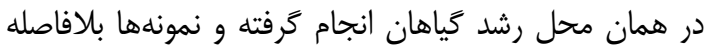
در مخزن ازت قرار داده شدند.
است كه قطعات برش يافته توسط آنزيمهاى برشى را بطور

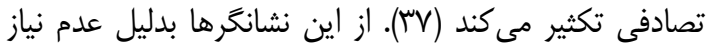

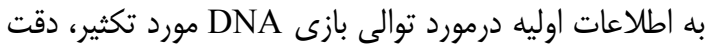

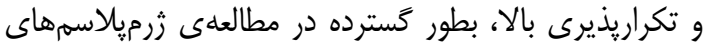

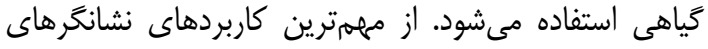

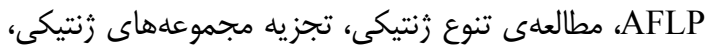

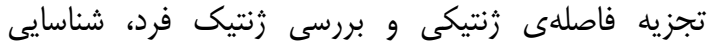

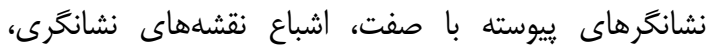

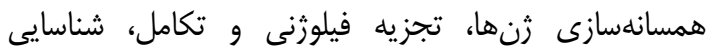

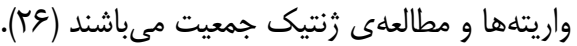

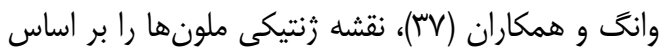

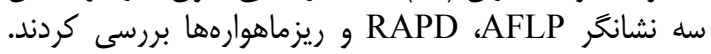

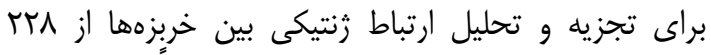

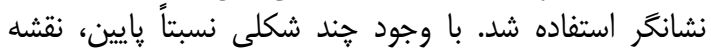

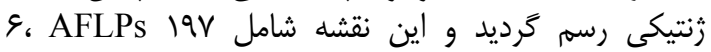

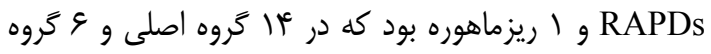

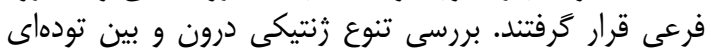

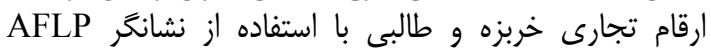

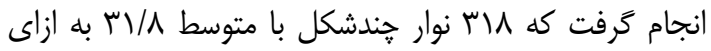

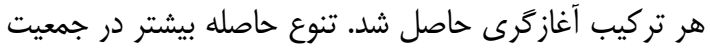

Table 1. Accession no. and Geographic location of wild melon genotypes

جدول 1 - كد و محل جغرافيايى نمونههاى خربزه وحشى

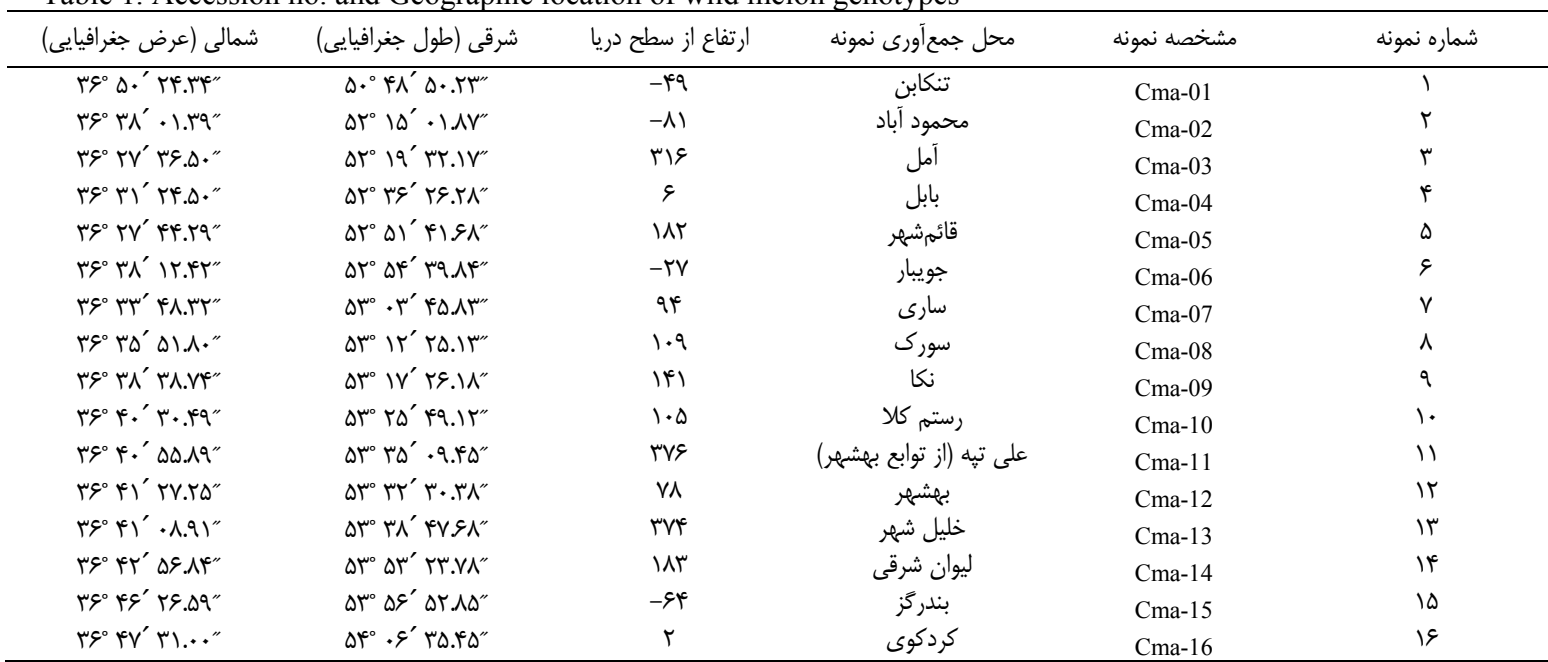

كلروفرم ايزوآميل -الكل به محلول اضافه كرده و به مانند قبل

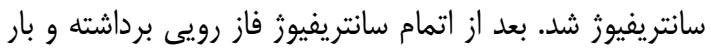

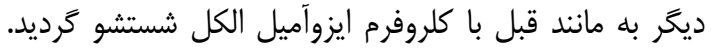

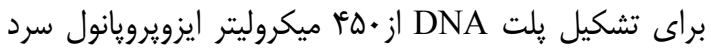

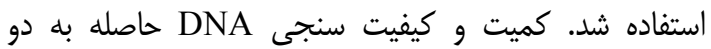

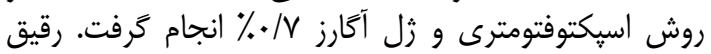

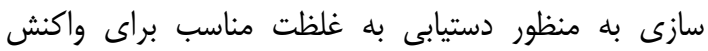
نيز با بر اساس غلظت دCR PCA استخراج شده صورت

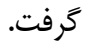

مراحل اجراى روش ترف

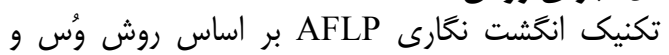

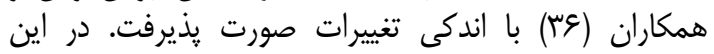

\section{استخراج استخراج}

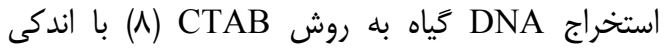

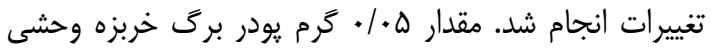

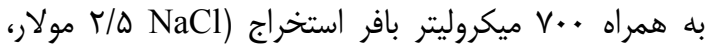
(w/v) إ مولار: Tris $\beta$ - $\quad r \%(w / v) \quad$ (PVP $\quad r \%(w / v) \quad$ ، CTAB $\quad \%$ Mercaptoethanol

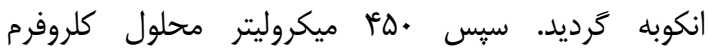

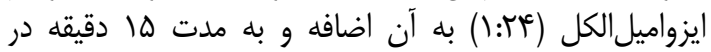

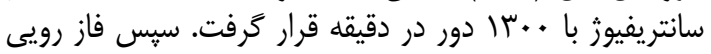

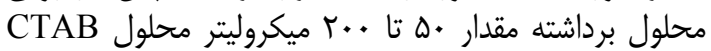

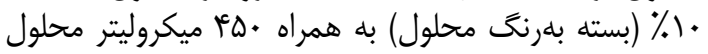




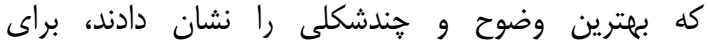

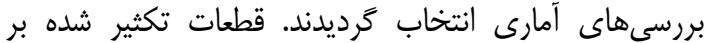

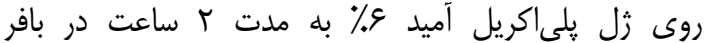

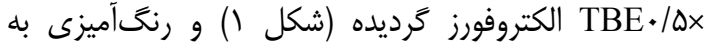

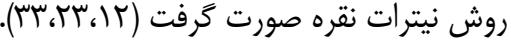

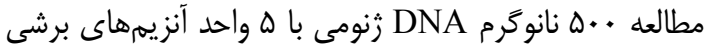

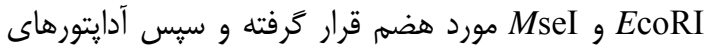

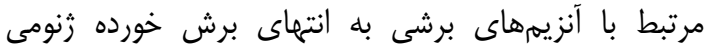

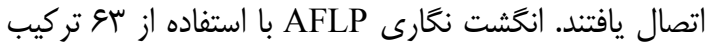

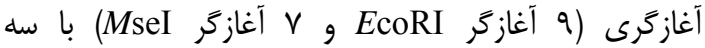

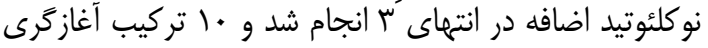

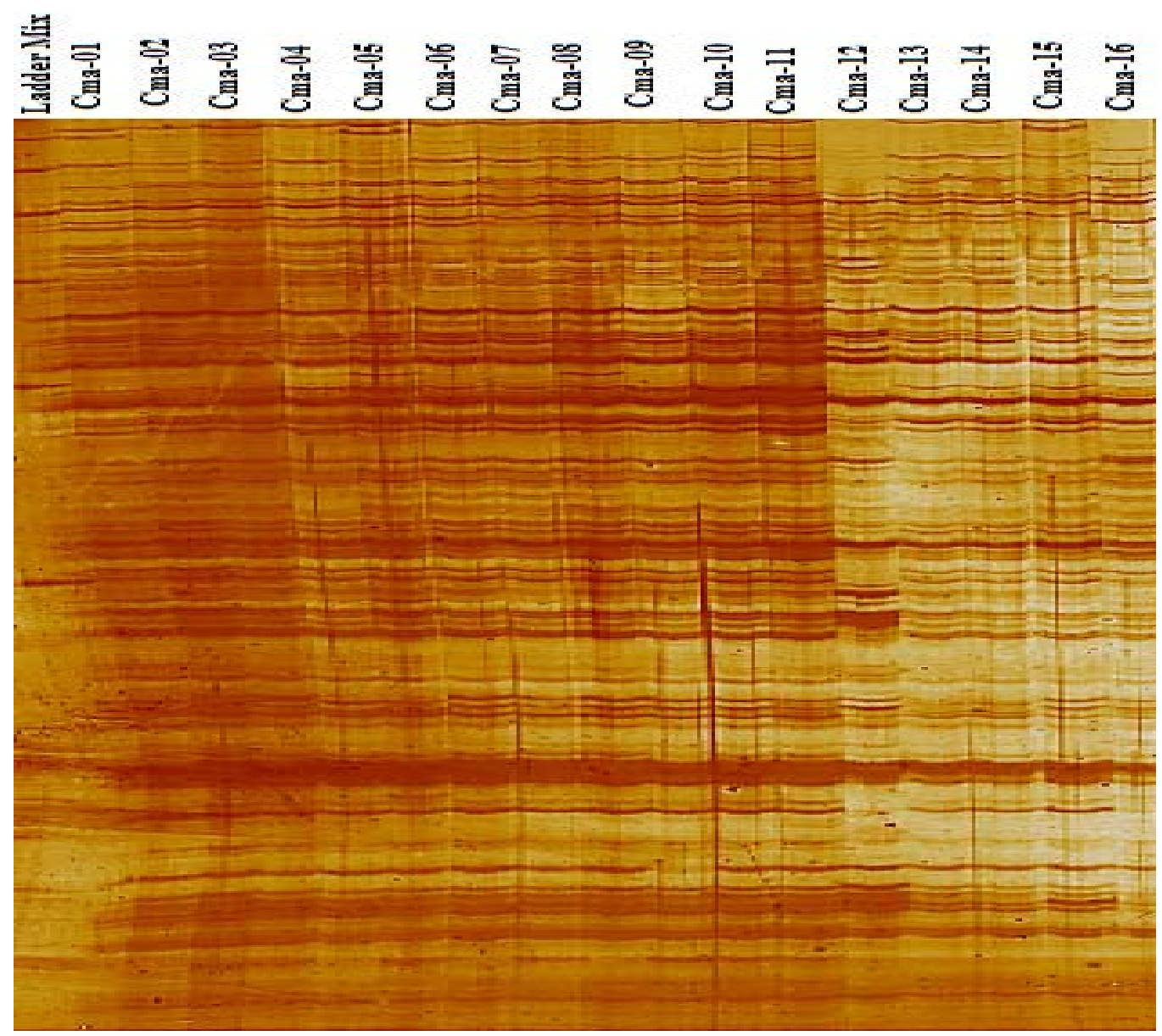

M-GAG/E-CCA نمونهاى خربزه وحشى بوسيله آغازگ AFLP شكل أ - الخوى هاى نوار

Figure 1. AFLP banding patterns with primer combinations M-GAG/E-CCA of wild melon genotypes

Win boot فاصله اطمينان 99 درصد بلوسيله نرمافزار جنار

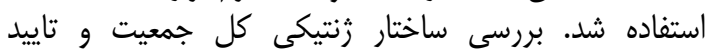

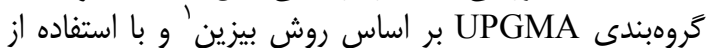

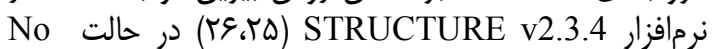

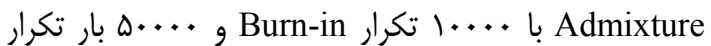
در مقادير مختلف KCMC

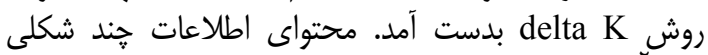

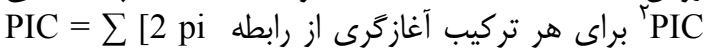

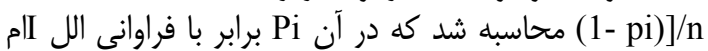

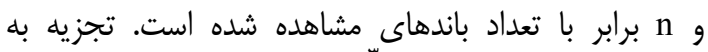

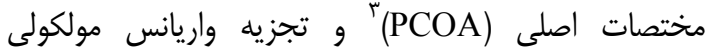
نيز با استفاده از نرمافزار 6.5 (AMOVA)

$$
\text { كرفت (r) }
$$

DNA الكتروفورز

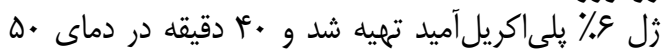

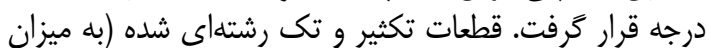

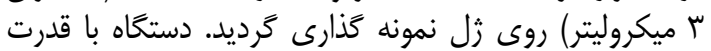

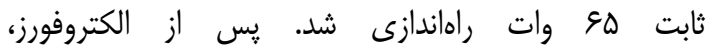

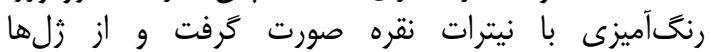
عكسبردارى شد. تجزيه دادهها

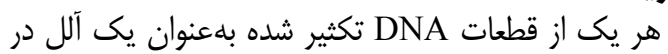

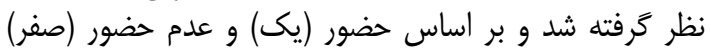

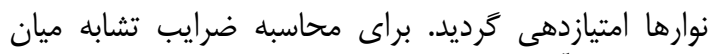

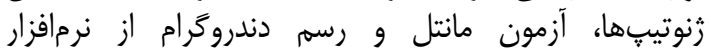
NTSYS 2.02

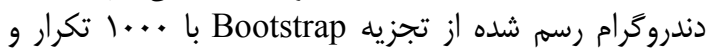


M- درصد بود. بيشترين ميزان קندشكلى را تركيب آغازگرى

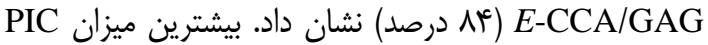
مربوط به نشانكر E-TCC/M-CCC و كمترين ميزان E-CTC مربوط به نشانكر E-CTC/M-GAG بود. متوسط مقدار در اين آزمايش عآ// • محاسبه كَرديد (جدول r).

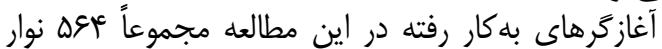

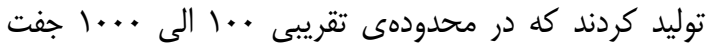

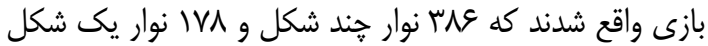

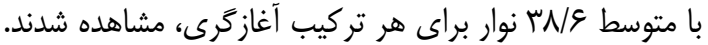
باطوركلى متوسط ميزان هندشكلى براى آغازكرها

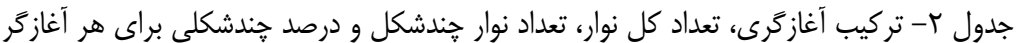
Table 2. Primer combinations, number of Total bands, number of polymorphic bands, polymorphism percentage of

\begin{tabular}{|c|c|c|c|c|}
\hline تركيب أغازكرى & تعداد كل نوار & تعداد نوارهاى جندشكل & درصد حندشكلى & PIC \\
\hline M-GAG, E-CCA & ru & rT & $\Lambda F$ &.$/ \kappa \Lambda$ \\
\hline M-AAG, E-СТА & $\Delta \Delta$ & is & 1) &.$/ T \Delta$ \\
\hline M-CCC, E-TCC & ५q & TV & 99 & א \\
\hline M-AAG, E-GTG & st & ఎ. & $\mathrm{vA}$ & $\cdot / T \Delta$ \\
\hline M-AAG, E-CTC & 99 & $\Delta r$ & VQ & .119 \\
\hline M-AAG, E-TCC & ז & rV & $\Delta \wedge$ & $\cdot / \pi \Delta$ \\
\hline M-GAG, E-CTC & $\Delta F$ & ז & $\Delta V$ & .19 \\
\hline M-CCC, E-CTC & at & io & v. & ع \\
\hline M-GAG, E-TCC & 99 & rV & $\Delta \Gamma$ &.$/ \mathrm{V}$ \\
\hline M-CCC, E-GTG & ir & rq & 9) & $\cdot / 1 \Lambda$ \\
\hline 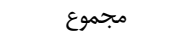 & $\Delta \& \varphi^{\circ}$ & ع או & & \\
\hline ميانكَين & $\Delta E / K^{c}$ & זN/ & SN/9 & $\cdot / \pi$ \\
\hline
\end{tabular}

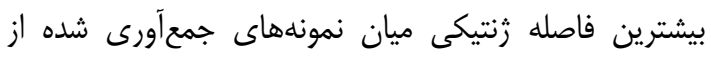

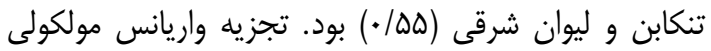

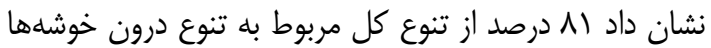

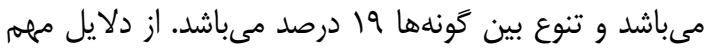

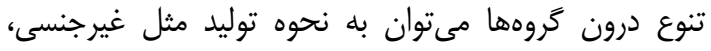

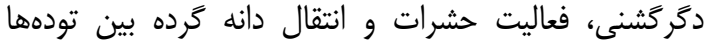

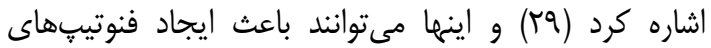

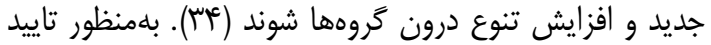

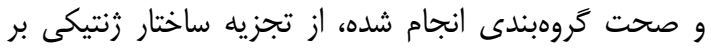
اساس روش بيزين با استفاده از نرمافزار

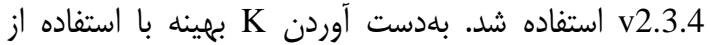

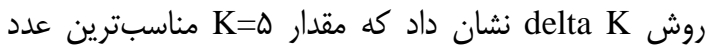

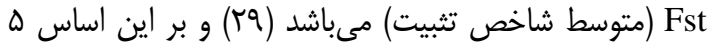

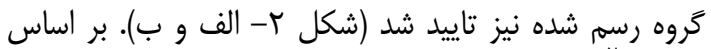

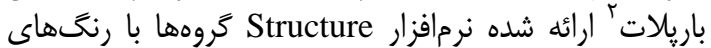

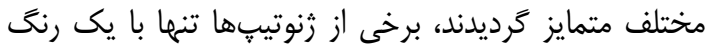

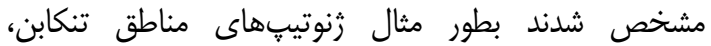

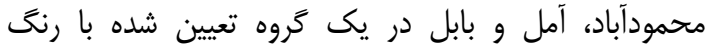

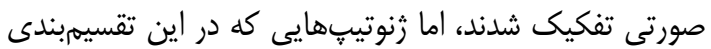

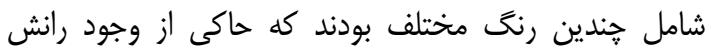

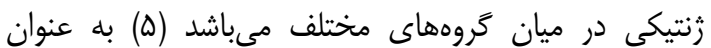

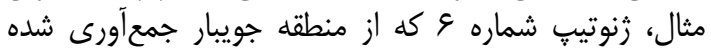

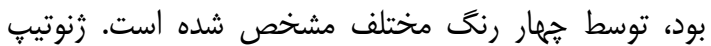

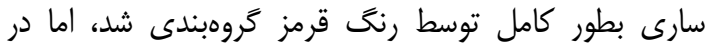

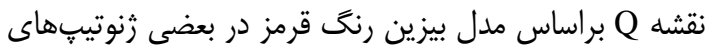

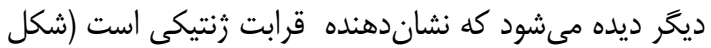

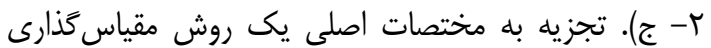

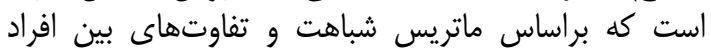
انجام مىشود و براى بوجود آوردن يك نمودار كرافرافيكى از بناز

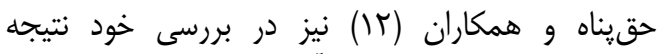

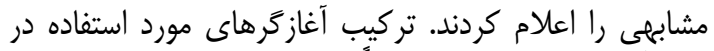

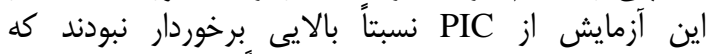

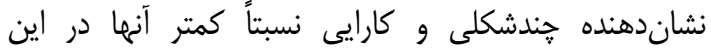

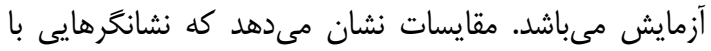

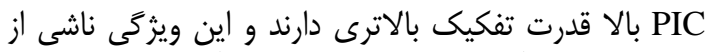

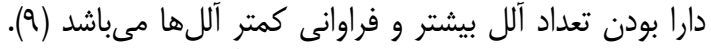

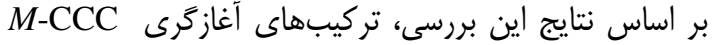

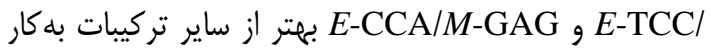

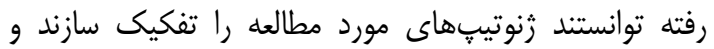
مىتوان از آنها در بررسى ثرد زئلاسم ساير ارقام و تودههاى

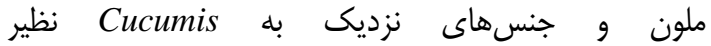
و Mymecosicyes ،Mukia ‘Dicaelopermum Oreosyce مورد آزمون نشان دادند كه بر اساس شاخص إنص شانون تركيب

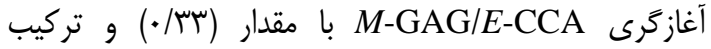
آغازگرى M-CCC/E-TCC

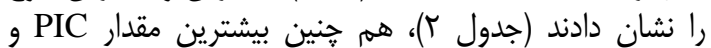

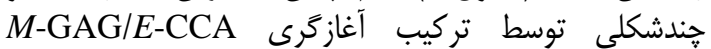

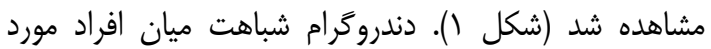

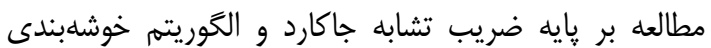
UPGMA

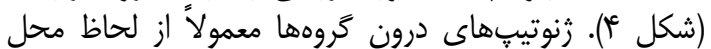

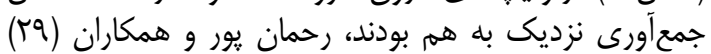

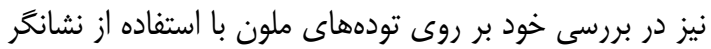

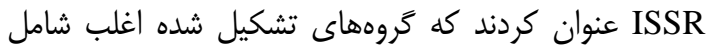

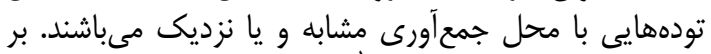
اساس ضريب تشابه جاكارد' بيشترين تشابه آنابه زنتيكى بين بين

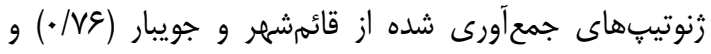




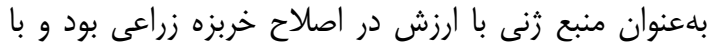

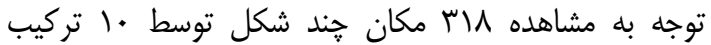

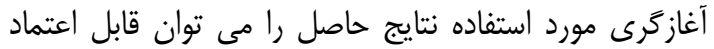

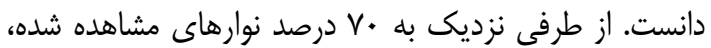

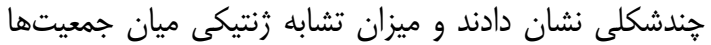

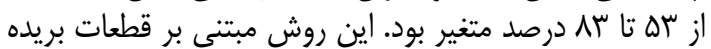

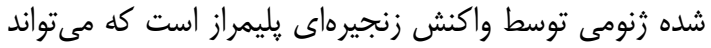

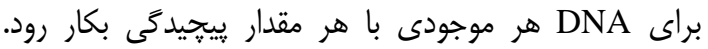

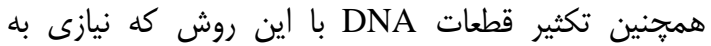

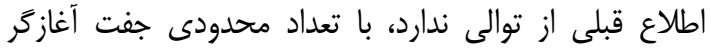

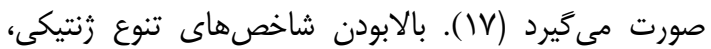

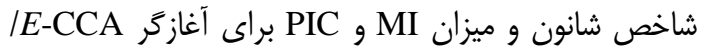
نشاندهنده كارايى بالاتر اين آغازكر در تمايز

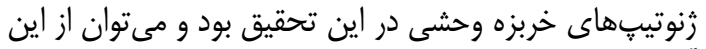

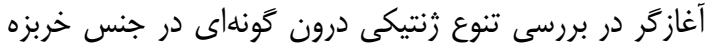

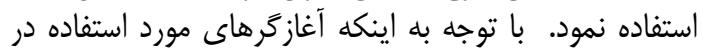

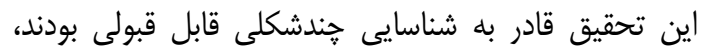

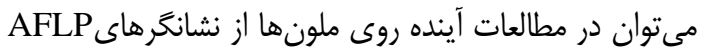

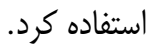

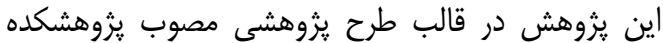

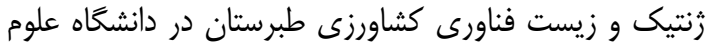

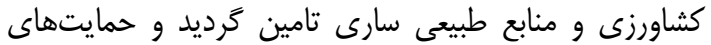

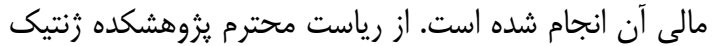

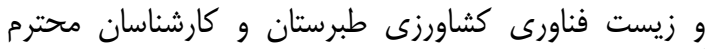

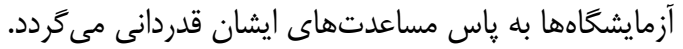

دادها با ابعاد كمتر هدف كذارى شده است (آس) بر اين اساس

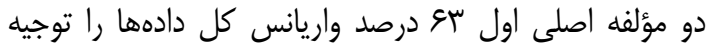

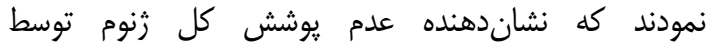

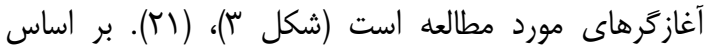

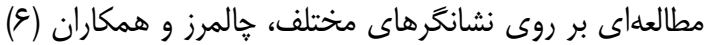

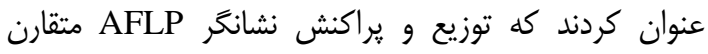

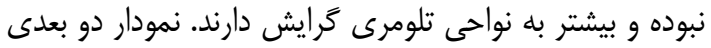

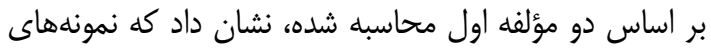

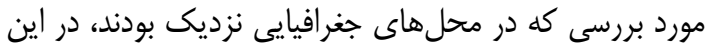

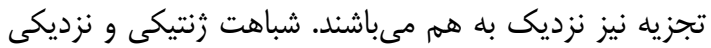

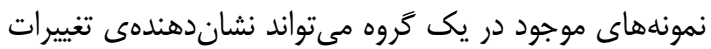

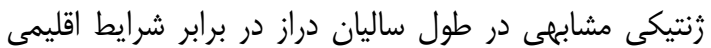

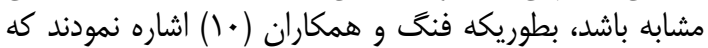

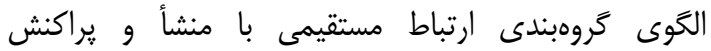

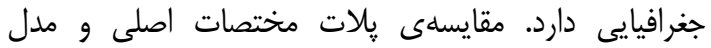

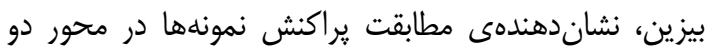

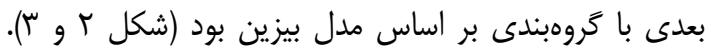

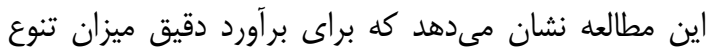

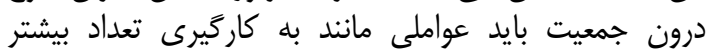

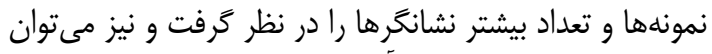

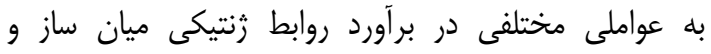

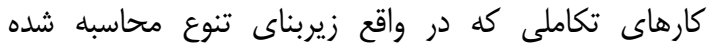

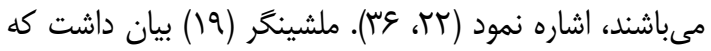

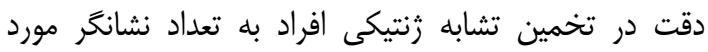

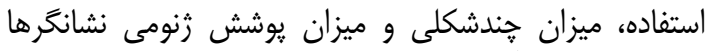

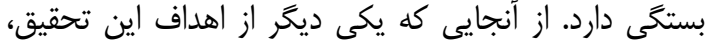

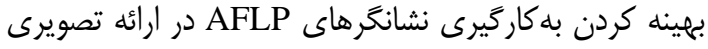

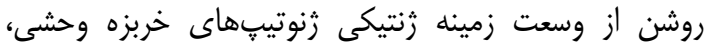

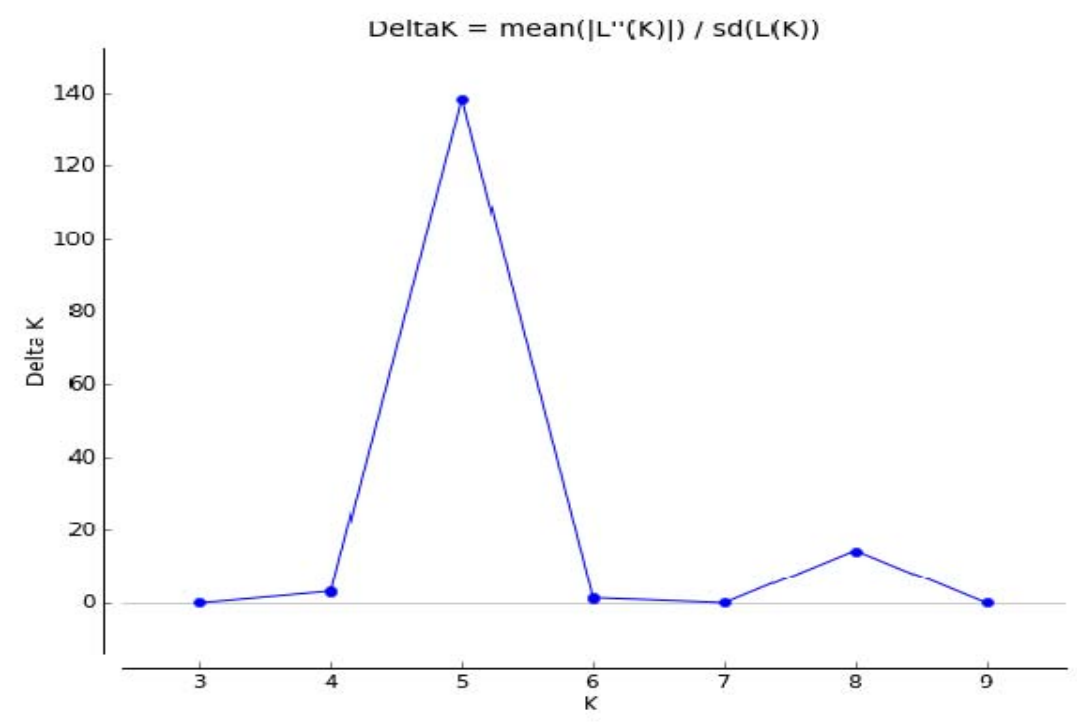

(الف) 
$(ب$

\begin{tabular}{|c|c|c|c|c|c|c|}
\hline $\mathrm{k}$ & Rep & MeanLnP(K) & $\operatorname{stdvLnP(K)}$ & $\operatorname{Ln}^{\prime}(\mathrm{K})$ & {$[\operatorname{Ln} "(\mathrm{~K})]$} & Delta K \\
\hline$r$ & $r$ & -DSVq/TGGGG & $F / K G G \mid Q \Lambda$ & - & - & - \\
\hline r & r & -QTrQ/..... & VE/VAFGTre & FDF/TGGG & $\mid$ | & - IIVGGAD \\
\hline f & r & - FVDV/ISGGG & 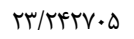 & 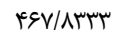 & $V \Delta / 1 \ldots$. & וזוIM/4 \\
\hline$\Delta$ & r & 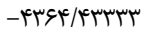 & $F+\Lambda \cdot V / 1 Q$ & משr/VTr & G. DQ/r\&q & ITN/TIQT \\
\hline$\varepsilon$ & r & -1..rM. & $\Delta \Lambda \| / M F \lambda+1$ & 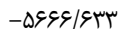 & MVYTr/FG & 1/Drga19 \\
\hline$\checkmark$ & r & 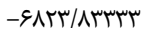 & rQqY/\&qFTD & سmr/rq.V & سזسو/ו"צو &.$/ K 11109$ \\
\hline 1. & r & -r.qr /A... & س & س & - & - \\
\hline
\end{tabular}

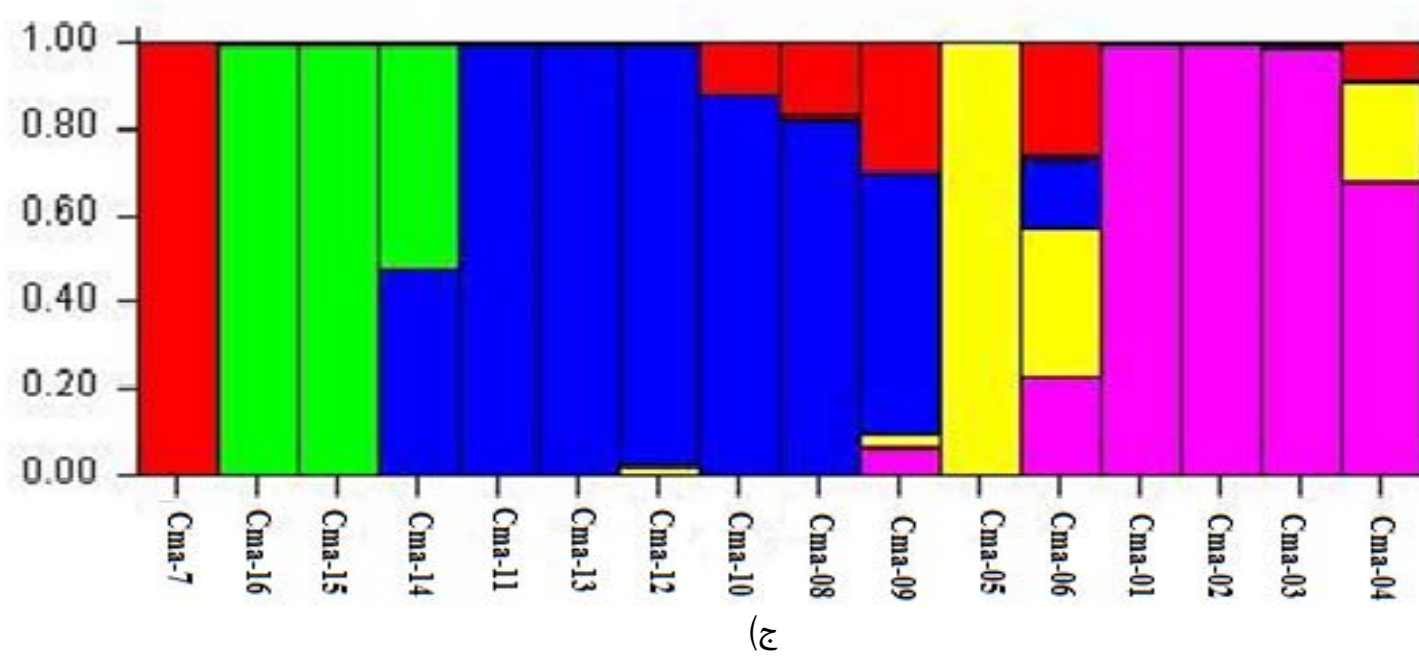

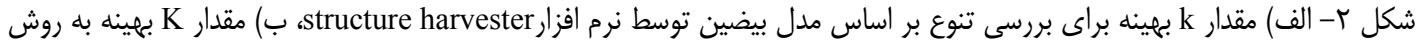
delta K AFLP

Figure 2. a) The optimum $\mathrm{K}$ for genetic diversity analysis based on Bayesian model, generated by structure harvester software, b) Optimum K based on delta K, c) Image of 16 wild melon genotypes based on Bayesian model $\mathrm{k}=5$

\section{Principal Coordinates (PCoA)}

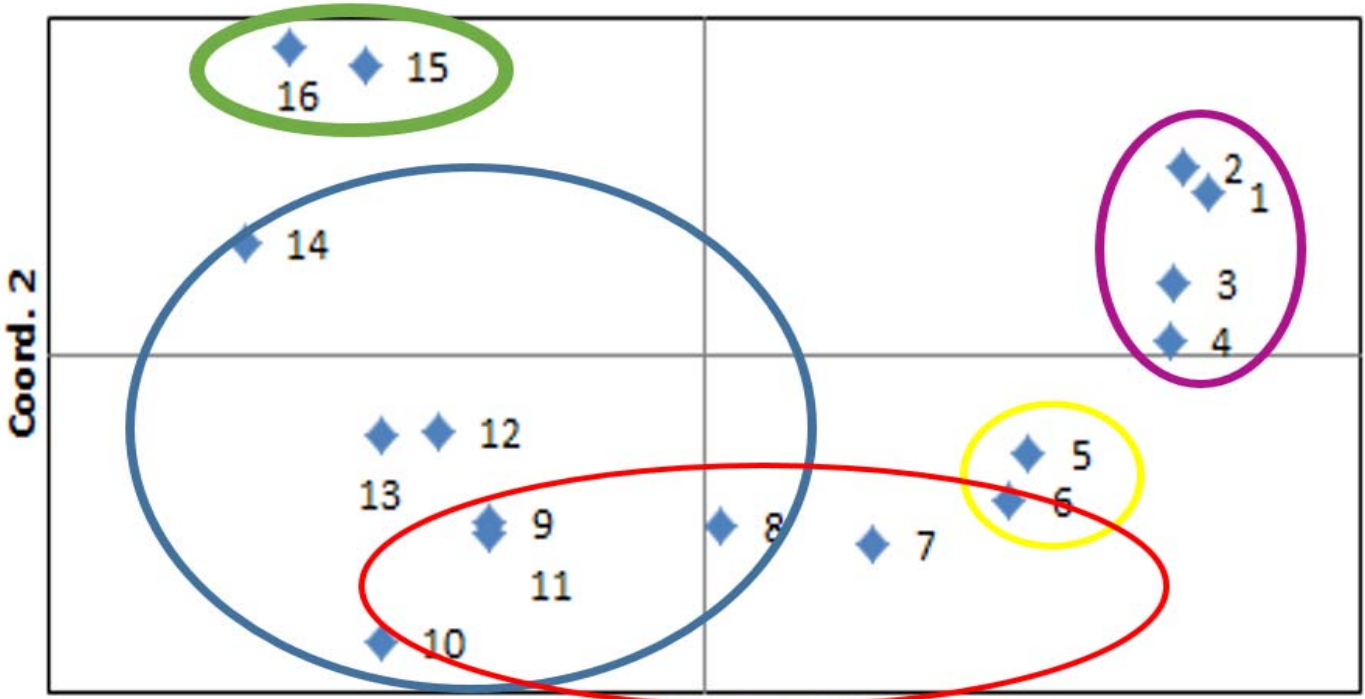

Coord. 1

شكل ب- رابطه ميان عا زنوتيّ مورد آزمون با استفاده PCoA

Figure 3. The relationship between 16 genotypes based on PCoA 


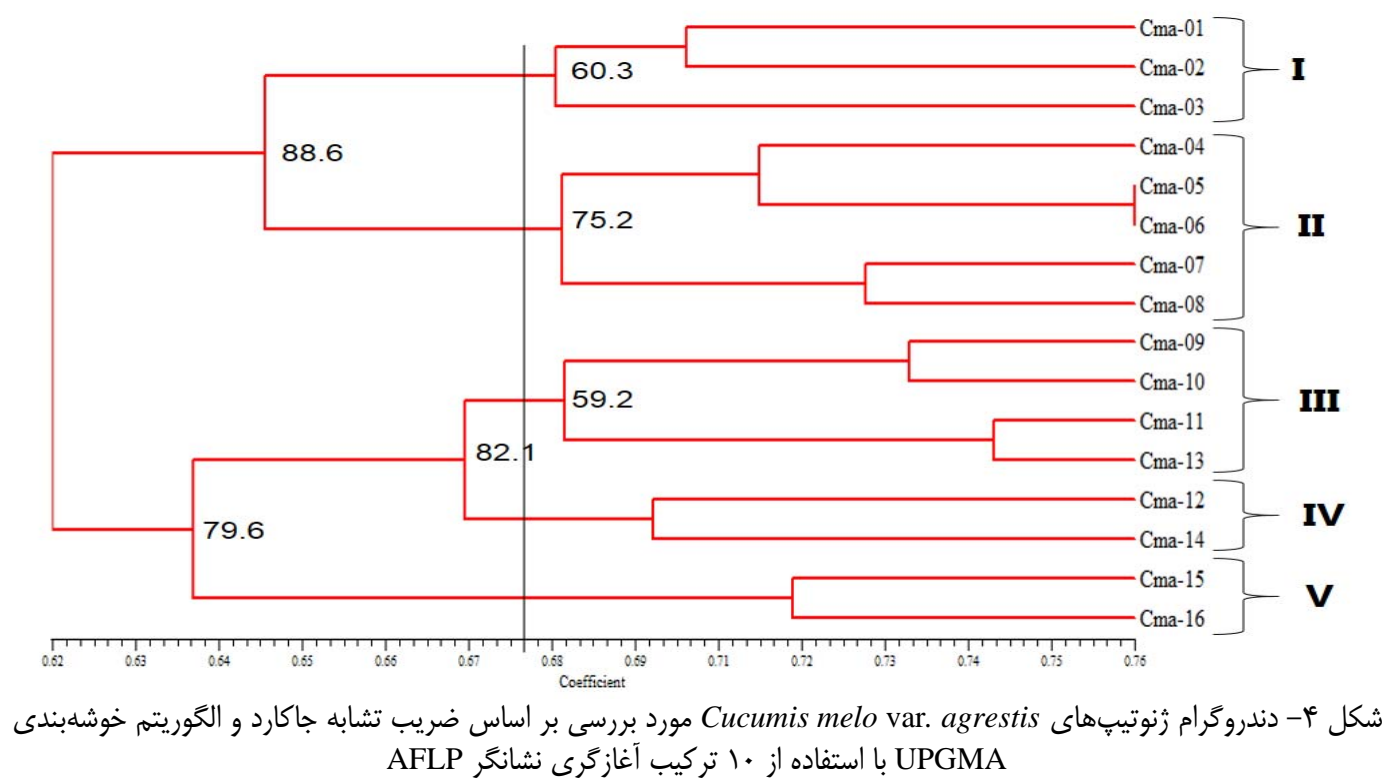

Figure 4. Cluster analysis of Cucumis melo var. agrestis based on Jaccard's similarity matrix and UPGMA algorithm using 10 AFLP primer combinations

1. Adekunle, A.A., O.A. Oluwo. 2008. The nutritive value of Cucumis melo var. agrestis Scrad (Cucurbitaceae) seeds and oil in Nigeria. American Journal of Food Technology, 3: 141-146.

2. Alagar Raja, M., G. Sahithi, R. Vasanthi, K.N.V. David Banji, and D. Selvakumar. 2015. Study of phytochemical and antioxidant activity of Cucumis melo var. agrestis fruit. Journal of Pharmacognosy and Phytochemistry, 2: 303-306.

3. Belja, A., Z. Satovic, G. Cipriani, L. Baldoni, R. Testolin, L. Rallo and I. Trujillo. 2003. Comparative study of the discriminating capacity of RAPD, AFLP and SSR Markers and of their effectiveness in establishing genetic relationships in olive. Theoretical and Applied Genetics, 107: 736 - 44.

4. Blancard, D., H. Lecoq, and M. Pitrat. 1994. A Colour Atlas of Cucurbit Diseases. Book: Manson Publishing, London, UK, 229 pp.

5. Carvalho, A., H. Guedes-Pinto, and J.E. Lima Brito. 2011. Genetic Diversity in old Portuguese durum wheat cultivars assessed by Retrotransposon-Based Markers. Plant Molecular Biology Reporter, 30: 578-589.

6. Chalmers, K.J., W. Canobell, J.A. Kretschmer, P.H. Enschke, S. Pierns., M. Harker, G.B. Pallota, M.R. Cornish, L. Shariflou, A. R.ampling, G. Mclauchlan, P.J. Daggard, T.A. Sharp, M. Holton, W. Sutherland, R. Apples and P. Landrige. 2001. Construction of three linkage maps in bread wheat, Triticum aestivum. Australian Journal Agriculture Research, 52: 1089-1119.

7. Danesh, M., M. lotfi, M. Naghavi, M. Pirseyedi and Kh. Nematallahi. 2007. Genetic diversity within and between mass Persian melon cultivars with markers AFLP. Proceedings of the National Conference and molecular biology, pp: 249-246 (In Persian).

8. Doyle, J.J. and J.L. Doyle. 1987. A rapid DNA isolation procedure for small quantities of fresh leaf tissue. Algal Physiology and Biochemistry, 19: 11-15.

9. Ebrahimi, A., M.R. Naghavi, M. Sabokdast and M. Mardi. 2010. Assessment of genetic diversity in two accessions of barely species $(H$. vulgare $L$. and $H$. Spontaneum L.) using SSR markers. Iranian Journal of Crop Sciences, 12: 333-345 (In Persian).

10. Feng, Z.Y., X.J. LIU, Y.Z. Zhang and H.Q. Ling. 2006. Genetic diversity analysis of Tibetan wild barley using SSR. Acta Genetica Sinica, 33: 917-928.

11. Garcia-Mas, J., M. Oliver, H. Gómez-Paniagua and M.C. de Vicente. 2000. Comparing AFLP, RAPD and RFLP markers for measuring genetic diversity in melon. Theoretical and Applied Genetics, 101: 860-864.

12. Haghpanah, H., S.K. Kazemitabar, S.H.R. Hashemi and S.M. Alavi. 2014. Evaluation of Mazandaran nettle (Urtica Dioica) population structure and genetic diversity by AFLP markers. Iranian Journal of Rangelands and Forests Plant Breeding and Genetic Research, 22: 241-250 (In Persian).

13. Hasani, M.H., S. Torabi, M. Omidi, A. Etminan and T. Dastmalchi. 2011. Assessment of genetic diversity of (Foeniculum vulgare Mill) using AFLP marker. Iranian Journal of Field Crop Science, 42: 594-604 (In Persian).

14. Heidari, S., H. Marashi, M. Farsi and A. Mirshamsi Kakhki. 2008. Genetic diversity in wild and cultivated populations of barberry (Berberis $s p$ ) from Khorasan using molecular markers AFLP. Journal of Horticultural Science, 22: 65-76 (In Persian). 
15. Jones, C.J., K.J. Edwards, S. Castsglione, M.O. Winfield and M. Matthes Daly. 1997. Reproducibility testing of RAPD, AFLP, SSR markers in plants by a network of European laboratories. Molecular Breeding, 3: 381-90.

16. Lima, M., A. Garcia, K. Oliveria, S. Matsuoka, H. Arizono and C.L. De Souza. 2002. Analysis of genetic similarity detected by AFLP and coefficient of parentage among genotypes of suger cane. Theoretical and Applied Genetics, 104: 30-38.

17. Mackill, D., E. Zhang, E. Redona and P. Colowtt. 1996. Level of polymorphism and genetic mapping of AFLP markers in rice. Genome, 39: 969-977.

18. Magiure, T.L., R. peakall and P. Saenger. 2002. Comparative analysis of genetic diversity in the managrove species Avicennia marina (Forsk) vierh. (Avicenniaceae) detected by AFLPs and SSRs. Theoretical and Applied Genetics, 93: 392-401.

19. Melchinger, A.E. and R.K. Gumber. 1999. Overview of hetrosis and hetrotic groups in agronomic crops in: K.R.Lamkay, J.E. Stuber (Eds) Concepts and breeding of heterosis in crop plants. Crop Science Society of America, 29-43 pp.

20. Mohammadi, S.A., M. Shokrpour, M. Moghaddam and A. Javanshir. 2011. AFLP-based molecular characterization and population structure analysis of Silybum marianum L. Plant Genetic Resources Characterization Utilization, 9: 445-453.

21. Mohammadi, S.A. and B.M. Prasanna. 2003. Analysis of genetic diversity in crop plants salient statistical tools and considerations. Crop Science, 43: 1235-1248.

22. Nei, M. 1978. Estimation of average heterozygosity and genetic distance from a small number of individuals. Genetics, 87: 583-590.

23. Saramirad, B., M. Shokrpour, O. Sofalian, A. Hasheminejad, E. Esfandiari and E. Esfandiari. 2016. Association Analysis of AFLP and RAPD Markers with Cadmium Accumulation in Wheat. Journal of Crop Breeding, 8: 126-133 (In Persian).

24. Saramirad, B., M. Shokrpour, O. Sofalian, A. Pourmohammad and E. Esfandiari. 2014. Evaluation of Genetic Diversity of Wheat Genotypes by AFLP Markers. Journal of Crop Breeding, 7: 89-96 (In Persian).

25. Pritchard, J.K., W. Wen and D. Falush. 2010. Documentation for STRUCTURE software Version 2.3. Department of Human Genetic University of Chicago, Department of Statistics University of Oxford.

http://pritch.bsd.uchicago.edu/structure_software/release_versions/v2.3.3/html/structure.html (Version 2.3.3)

26. Pritchard, J.K., M. Stephens and P. Donnelly. 2000. Inference of population structure using multilocus genotype data. Genetics, 155: 945-95.

27. Pejic, I., M.P. Ajmone and M. Morgante. 1998. Comparative analysis of genetic similarity among maize inbred lines detected by RFLPS, RAPDs, SSRs, and AFLPs. Theoretical and Applied Genetics, 97: 1248-1255.

28. Powell, W., M. Morgante, C. Andre, J. Vogel, S. Tingey and A. Rafalski. 1996. The comparison of RFLP, RAPD, AFLP and SSR (microsatellite) markers for germplasm analysis. Molecular Breeding, 2: 225-238.

29. Rahmanpour, S., B. Abdollahi Mandoulakani and M. Ghadimzadeh. 2014. Assessment of genetic diversity in Iranian melon (Cucumis melo L.) landraces and hybrids using ISSR markers. Modern Genetics. 9: 67-76 (In Persian).

30. Renner. S.S.. H. Schaefer and A. Kocvan. 2007. Phvlogenetics of Cucumis (Cucurbitaceae) Cucumber (C. sativus) belongs in an Asian/Australian clade far from melon (C. melo). BMC Evolutionary Biology, 7: 58.

31. Rohlf, F.J. 1972. An empirical comparison of three ordination techniques in numerical taxonomy. Systematic Zoology, 21: 271-280.

32. Roy, A., S.S. Bal, M. Fergany, S. Kaur, H. Singh, A. Malik, J. Singh, A.J. Monforte and N.P.S. Dhillon. 2012. Wild melon diversity in India (Punjab State). Genetic Resources and Crop Evolution, 59: 755-767.

33. Sanguinetti, C.J., E. Dias Neto and A.J.G. Simpson. 1994. Rapid silver staining and recovery of PCR products separated on polyacrylamide gels. Bio techniques, 17: 915-919.

34. Shi, W., C.F. Yang, J.M. Chen and Y.H. Guo. 2008. Genetic variation among wild and cultivated populations of the Chinese medicinal plant Coptis chinensis (Ranunculaceae). Plant Biology, 10: 485-491.

35. Talebi Kohyakhy, E., M.R. Naghavi and M. Mohammad Aliha. 2008. Study of the essential oil variation of Ferula gummosa samples from Iran. Chemistry of Natural Compounds, 44: 124 -126 (In Persian).

36. Vos, P., R. Hogers, M. Bleeker, M. Reijans, T.V.D. Lee, M. Hornes, A. Frijters, J. Pot, J. Peleman, M. Kuiper and M. Zabeau. 1995. AFLP: a new technique for DNA fingerprinting. Nucleic Acids Research, 23: 4407-4414.

37. Wang, Y.H., C.E. Thomas and R.A. Dean. 1997. A genetic map of melon (Cucumis melo L.) based on amplified fragment length polymorphism (AFLP) marker. Theoretical and Applied Genetics, 95: 791-798. 


\title{
Assessment of Genetic Diversity and Structure in the Wild Melon (Cucumis melo var. agrestis) Genotypes from Southern Coastline of Caspian Sea using AFLP Markers
}

\author{
Fatemeh Vafadar Shamasbi ${ }^{1}$, Ali Dehestani ${ }^{2}$, Saber Golkari ${ }^{3}$ and Mostafa Haghpanah ${ }^{4}$ \\ 1- Department of Agricultural Biotechnology, Maragheh Branch, Islamic Azad University, Maragheh, Iran \\ 2- Genetics and Agricultural Biotechnology Institute of Tabarestan, Sari Agricultural Sciences and Natural \\ Resources University, Sari, Iran (Corresponding Author: a.dehestani@ sanru.ac.ir) \\ 3- Dryland Agriculture Research Institutes (DARI), Agricultural Research, Education and Extension Organisation \\ (AREEO) Maragheh, Iran \\ 4- Department of Biotechnology and Plant Breeding, Sari Agricultural Sciences and Natural Resources \\ University, Sari, Iran \\ Received: June 18, $2015 \quad$ Accepted: December 22, 2015
}

\begin{abstract}
Wild melon (Cucumis melo var. agrestis) commonly known as African melon grows in tropical and semi tropical regions of the world. It also known as a valuable medicinal plant has numerous pharmaceutical properties and is considered as a valuable germplasm in cultivated melons breeding. In the present study, genetic variation of sixteen wild melons, collected from Mazandaran province, was examined by 10 AFLP markers. In total, 564 bands were generated and more than 68\% were polymorphic. Mean Polymorphic Information Content (PIC) and Marker Index (MI) values were 0.22 and 15.51 , respectively and primer $(E-C C A / M-C A G)$ indicated the highest MI, accounting 24.19. In addition, primer $(E-C C A / M-C A G)$ showed the highest diversity with a Shannon Index 0.33. Cluster analysis using Jaccard similarity method and UPGMA algorithm, and Bayesian method, grouped all genotypes in five main groups. Results of Principal Coordinate Analysis were similar with the results based on Bayesian model and the cluster analysis. Genetic diversity determined based on AFLP markers confirmed the efficiency of AFLP for classifying wild melon genotypes according to the geographical origins and such markers can be used to identify differences within species in the genealogy studies.
\end{abstract}

Keywords: Amplified Fragment Length Polymorphism (AFLP) marker, Bayesian method, Genetic Diversity, Wild melon 